\title{
Tangence
}

\section{Du matérialisme à l'immatérialisme : le problème âme-corps dans la philosophie clandestine}

\section{Sébastien Charles}

Numéro 81, été 2006

Libertinage et clandestinité à l'âge classique

URI : https://id.erudit.org/iderudit/014964ar

DOI : https://doi.org/10.7202/014964ar

Aller au sommaire du numéro

Éditeur(s)

Tangence

ISSN

1189-4563 (imprimé)

1710-0305 (numérique)

Découvrir la revue

Citer cet article

Charles, S. (2006). Du matérialisme à l'immatérialisme : le problème âme-corps dans la philosophie clandestine. Tangence, (81), 143-161.

https://doi.org/10.7202/014964ar
Résumé de l'article

Parmi l'ensemble des manuscrits philosophiques clandestins à notre disposition, la grande majorité des textes adopte une position matérialiste quant au rapport entre l'âme et le corps. Cette position est souvent défendue à partir d'une critique du dualisme cartésien dont on mentionne les difficultés qui ressortissent à l'union improbable de deux substances ontologiquement différentes. Mais cette lecture n'épuise pas l'ensemble des réactions nées des difficultés du dualisme cartésien. À cet égard, l'étude des Réflexions morales et métaphysiques sur les connaissances de l'homme permet de complexifier l'analyse. De fait, ce manuscrit cherche à tracer une voie médiane entre matérialisme et idéalisme d'où découle une métaphysique proprement originale, cherchant à réconcilier l'idéalisme de Malebranche et le panthéisme de Spinoza, et témoignant par là de la richesse et de la nouveauté de certaines réflexions clandestines. 


\title{
Du matérialisme à l'immatérialisme : le problème âme-corps dans la philosophie clandestine
}

\author{
Sébastien Charles, \\ Université de Sherbrooke
}

\begin{abstract}
Parmi l'ensemble des manuscrits philosophiques clandestins à notre disposition, la grande majorité des textes adopte une position matérialiste quant au rapport entre l'âme et le corps. Cette position est souvent défendue à partir d'une critique du dualisme cartésien dont on mentionne les difficultés qui ressortissent à l'union improbable de deux substances ontologiquement différentes. Mais cette lecture n'épuise pas l'ensemble des réactions nées des difficultés du dualisme cartésien. À cet égard, l'étude des Réflexions morales et métaphysiques sur les connaissances de l'homme permet de complexifier l'analyse. De fait, ce manuscrit cherche à tracer une voie médiane entre matérialisme et idéalisme d'où découle une métaphysique proprement originale, cherchant à réconcilier l'idéalisme de Malebranche et le panthéisme de Spinoza, et témoignant par là de la richesse et de la nouveauté de certaines réflexions clandestines.
\end{abstract}

Depuis une vingtaine d'années, la recherche sur les manuscrits philosophiques clandestins de l'âge classique a connu un véritable renouveau et les nombreuses études publiées depuis lors permettent de se faire une idée de plus en plus appropriée de cet objet d'étude problématique. Au fil des recherches et des publications des résultats de celles-ci ces dernières années, une image plutôt matérialiste et naturaliste s'est dégagée de l'ensemble des manuscrits, mais cet aspect n'épuise pas la totalité des textes qui sont à notre disposition. Ainsi, concernant le statut de l'âme humaine, à propos duquel on peut penser qu'une lecture matérialiste prédomine, certains manuscrits prennent le contre-pied de la thèse avancée par la grande majorité des auteurs clandestins. Si l'on s'en tient donc à la question très discutée à la fin du XVII siècle et au début du XVIII ${ }^{\mathrm{e}}$ siècle des rapports entre l'âme et le corps, on se doit 
de reconnaître que cette problématique est effectivement dominée dans les manuscrits clandestins par une conception naturaliste ou matérialiste de ce rapport, conception qui a fait du cartésianisme son adversaire déclaré. Mais on se doit également de concéder que cette uniformité n'est pas absolue, comme nous le montrerons en nous attachant à l'étude d'un manuscrit quelque peu singulier, qui tranche vivement sur cette question avec l'ensemble plutôt homogène de la clandestinité philosophique.

\section{La critique du cartésianisme}

Si l'on trouve dans les manuscrits clandestins de la fin du $\mathrm{XVII}^{\mathrm{e}}$ siècle et du début du XVIII ${ }^{\mathrm{e}}$ siècle de nombreuses traces du naturalisme ancien et renaissant ${ }^{1}$, les auteurs de ces manuscrits n'en ont pas moins conscience que leurs critiques portent contre un adversaire nouveau, qui a redéfini à son profit la question. Comme l'écrit Mirabaud, à qui l'on attribue le manuscrit De l'âme et de son immortalité, "aujourd'hui on entend généralement par le mot âme une substance immatérielle tellement unie au corps, que les mouvements de l'un sont nécessairement suivis des mouvements de l'autre ${ }^{2} »$. Confirmation en est donnée dans l'histoire que l'on propose de l'âme, sujet trivial dans bon nombre de manuscrits clandestins, où l'on montre que les Anciens l'ont généralement conçue matérielle et pensée sous la forme d'un souffle ou d'une matière subtile, à l'exception des pythagoriciens et des platoniciens, et où l'on présente une généalogie précise de l'âme permettant d'expliquer comment s'est dégagée peu à peu une notion de cette substance qui en fait un principe spirituel absolument différent du corps chez les cartésiens. La première salve de critiques porte contre le caractère spirituel de l'âme, mais sans en rester là. Car, au fait des débats internes et externes au cartésianisme, les auteurs clandestins savent pertinemment que la spiritualité de l'âme évoquée par les cartésiens conduit à deux autres conséquences non négligeables: 1) l'âme étant spirituelle, elle est

1. Sur la question des influences qui sont manifestes à l'égard du problème âmecorps chez les auteurs clandestins et déistes européens, voir l'article très complet de Giuseppe Ricuperati, «Il problema della corporeità dell'anima dai libertini ai deisti», dans Sergio Bertelli (sous la dir. de), Il libertinismo in Europa, Milan et Naples, Ricardo Ricciardi, 1980, p. 368-415.

2. [Jean-Baptiste de Mirabaud], "De l'âme et de son immortalité", dans Naigeon, Encyclopédie méthodique, Paris, H. Agasse, 1795, vol. III, p. 293 b. 
donc conçue comme incorruptible, ce qui a comme conséquence de la rendre immortelle; 2) l'âme étant spirituelle, elle est ce qui permet de distinguer de manière adéquate l'homme de l'animal. Voyons donc brièvement le traitement réservé à ces trois thèses cartésiennes - spiritualité de l'âme, immortalité de l'âme, conception de l'animal-machine - dans les manuscrits clandestins.

Le manuscrit De l'âme et de son immortalité a l'avantage de résumer sous une forme syllogistique ce qui passe pour être l'argumentation de Descartes en faveur de la spiritualité de l'âme: «La matière est incapable de penser; or il y a en moi quelque chose qui pense; donc il y a en moi une substance différente de la matière, et c'est ce que j'appelle l'esprit ${ }^{3}$.» Pour Mirabaud, la majeure du syllogisme pose problème, car elle apparaît trop catégorique. Il semble en effet que nous ne connaissions pas toutes les propriétés de la matière, et encore moins la nature exacte de la pensée comme le prétend Descartes. Comment alors en conclure de manière aussi catégorique au fait que la matière est incapable de penser? C'est là un leitmotiv de la pensée clandestine qui reprend à son compte, et ce bien avant Voltaire, l'indication lockienne de la possibilité d'une matière pensante et la critique des idées innées afin de réduire la pensée, et par là l'âme, à une modification de la matière.

Descartes commettrait donc une faute logique en restreignant l'essence de la matière à l'étendue. Comme l'avance l'Origine des êtres et espèces, manuscrit que l'on attribue à Boulainviller, "les propriétés de la matière ne sont pas assez connues, et comme il n'y a nul rapport de la pesanteur d'un corps à sa couleur, et que même un aveugle n'aurait nulle idée de la dernière, on pourrait nier que ces propriétés pussent subsister en un même sujet, et on le nierait mal à propos ${ }^{4} »$. Même idée dans Parité de la vie et de la mort: "Je me représente ici Descartes comme un homme qui se rappellerait l'idée du cuivre, qui saurait qu'il est fusible, malléable et propre à mettre en limaille; mais qui cependant, sur l'idée de ses propriétés, nierait qu'il fut capable de marquer les heures, par la seule raison

3. [Jean-Baptiste de Mirabaud], «De l'âme et de son immortalité», ouvr. cité, p. 313a.

4. [Henri de Boulainviller], "Origine des êtres et espèces, fruit d'une conversation retenue imparfaitement» [1705-1710], dans G. Mori et A. Mothu (sous la dir. de), Philosophes sans Dieu. Textes athées clandestins du XVIII siècle, Paris, Honoré Champion, 2005, p. 48. 
qu'il ne conçoit pas que du cuivre puisse produire un tel effet ${ }^{5}$.» Après tout, comme l'évoque l'auteur de L'âme matérielle, Newton vient tout juste de découvrir une nouvelle propriété de la matière, qui est l'attraction naturelle des corps à distance. Pourquoi serait-il impossible dans l'avenir de démontrer que la pensée est une autre propriété essentielle de la matière? Sur une question aussi délicate, il est plus salutaire de suspendre son jugement, comme le propose l'auteur des Doutes des pyrrhoniens dans son quatrième doute ${ }^{6}$, ou d'invoquer les bornes de notre connaissance que d'agir avec précipitation et de conclure sans savoir.

L'autre problème que pose la thèse de la spiritualité de l'âme opposée à la matérialité du corps est celle du rapport entre deux substances ainsi différenciées au plan ontologique et du lieu exact de leur rencontre, critiques déjà formulées en son temps par Gassendi ${ }^{7}$. Comme le voit bien l'auteur clandestin des Essais sur la recherche de la vérité, la spiritualisation de l'âme pose aussi le problème de sa localisation dans le corps, que ne résout pas l'hypothèse de la glande pinéale ${ }^{8}$, question qui est par contre résolue si

5. Parité de la vie et de la mort. La réponse du médecin Gaultier, édition d'Olivier Bloch, Oxford et Paris, The Voltaire Foundation et Universitas, 1993, p. 249250 (voir aussi p. 98). On trouve un exemple analogue dans Dissertation sur la formation du monde [1738], Paris, Champion, 2001, p. 121: "Lorsque Descartes a conclu que la matière ne pouvait produire le sentiment dans les êtres qu'elle modifiait, parce qu'il n'apercevait point de sentiment dans les particules de la matière, il a agi comme un homme qui, considérant les pièces éparses d'un moulin, soutiendrait que la pierre et le bois ne sauraient acquérir la qualité de moudre le grain; en effet, aucune de ces pièces seule ne peut moudre, mais réunies elles acquièrent de nouvelles qualités, et écrasent et pulvérisent le grain. D'où il résulte que les effets, tels que le mouvement et le sentiment, ne viennent que de l'enchaînement et de la correspondance, et de l'action unanime des pièces assorties dans les êtres. "

6. Sur les Doutes des pyrrhoniens, voir les deux articles que lui a consacrés Gianni Paganini, «Scepsi clandestina: i Doutes des pyrrhoniens», dans G. Canziani (sous la dir. de), Filosofia e religione nella letteratura clandestina, Milan, Franco Angeli, 1994, p. 83-122; et «Du bon usage du scepticisme: les Doutes des pyrrhoniens", dans Antony McKenna et Alain Mothu (sous la dir. de), La philosophie clandestine à l'âge classique, Oxford et Paris, The Voltaire Foundation et Universitas, 1997, p. 291-306.

7. Gassendi reprend à son compte, dans la Disquisitio metaphysica (400b), l'argument développé par Lucrèce dans son De rerum natura (I, 304) : «toucher et être touché ne peut être le fait que d'une chose corporelle».

8. Comme en témoigne également l'auteur de L'âme matérielle qui, s'inspirant de Bayle et de La Forge, voit bien que «si l'on dit avec les Cartésiens que l'âme n'est pas étendue par tout le corps, mais qu'elle n'occupe qu'un lieu: soit que ce lieu soit la glande pinéale, comme l'a cru le rêveur Descartes, soit que ce 
l'on pense ces deux substances comme matérielles: «Je ne puis m'empêcher de trouver fort extraordinaire qu'on ait agité cette même question, de la place que doit occuper l'âme, lors même qu'on la supposait spirituelle; car il semble que rien n'implique tant de contradiction que de dire que l'âme est spirituelle et de vouloir en même temps qu'elle réside quelque part, et surtout dans un lieu particulier ${ }^{9}$.»

Peut-on évoquer l'hypothèse occasionnaliste pour sauver la spiritualité de l'âme? L'auteur clandestin de L'âme matérielle invoque Leibniz à son secours pour montrer que cette thèse suppose des miracles perpétuels, Dieu étant «sans cesse occupé à mouvoir sa machine lui-même et à en faire jouer les ressorts ${ }^{10}$ ", qu'elle fait de Dieu l'auteur du péché et qu'elle fournit au final une bien mauvaise image du Créateur: "On a des sentiments bien bas de la puissance et de la sagesse de Dieu s'il n'a pu faire l'homme sans être obligé d'agir à sa place à tout moment; c'est étrangement avilir la divinité que de la faire intervenir de la sorte, et il était inutile de faire une aussi admirable machine que le corps, si toute cette belle disposition ne sert de rien du tout pour porter les sensations jusqu'à l'esprit ${ }^{11}$.»

Ainsi, puisque l'incommunicabilité foncière entre substances matérielle et spirituelle paraît établie, il semble plus évident aux critiques de l'âme spirituelle de s'en tenir à une interaction entre âme matérielle et corps étendu fondée sur une physiologie intégralement mécaniste: ce sont les organes des sens qui agissent sur les esprits animaux et qui font naître dans l'âme les perceptions et les sentiments de plaisir ou de douleur. La notion de spiritualité leur paraît ne rien expliquer et être, selon l'expression du curé Meslier, une "idée sans modèle ${ }^{12}$ ", c'est-à-dire un concept non opératoire

soit quelque partie du corps, comme le prétendent les mêmes Cartésiens, la même difficulté revient toujours, puisque l'âme ne peut pas occuper un lieu tel qu'on se le figure, qu'elle ne soit étendue et par conséquent qu'elle n'ait des parties» (édition d'Alain Niderst, Paris, Presses universitaires de France, 1973, p. 62-64).

9. Essais sur la recherche de la vérité, dans G. Mori et A. Mothu (sous la dir. de), Philosophes sans Dieu. Textes athées clandestins du XVIII siècle, ouvr. cité, p. 241.

10. L'âme matérielle, ouvr. cité, p. 60.

11. L'âme matérielle, ouvr. cité, p. 58.

12. Le bon sens du curé Meslier, Paris, Garnier frères, 1905, p. 49. Meslier critique également la notion de simplicité: "On nous assure que l'âme humaine est une substance simple; mais si l'âme est une substance si simple, elle devrait être précisément la même dans tous les individus de l'espèce humaine, qui 
que l'auteur des Réflexions sur l'existence de l'âme et sur l'existence de Dieu, en digne successeur d'Occam, nous invite à raser ${ }^{13}$. À l'inverse, le concept de matière peut être conservé puisque nos sens nous en démontrent et l'existence et l'action. Ainsi, toutes les opérations de la pensée peuvent s'expliquer en termes d'organisation et de constitution matérielle, comme Condillac tâchera par la suite de le démontrer à l'aide du fameux exemple de la statue animée. Pour les clandestins, l'expérience semble trancher en faveur de leur hypothèse matérialiste. On voit en effet que les accidents qui affectent le corps, et en particulier le cerveau, affectent également l'âme (lésion cérébrale, abus d'alcool, maladies, influence des passions, âges de la vie, etc.), ce qui permet de conclure à leur identité substantielle. Au fond, si l'on ne se résout pas à la matérialité de l'âme, c'est d'abord, au plan personnel, par amourpropre, c'est-à-dire par souci de se différencier des autres êtres vivants et par refus de se penser mortel, et ensuite, au plan collectif, afin de tenir les citoyens en respect en les forçant à bien se conduire par la crainte de châtiments éternels.

La question de l'immortalité de l'âme permet une nouvelle confrontation avec les cartésiens qui ont tiré de la différence ontologique entre res cogitans et res extensa une preuve en faveur de l'incorruptibilité de l'âme, renouvelant par là le traitement habituel du problème ${ }^{14}$ qui reposait en général sur l'argument du

tous devraient avoir les mêmes facultés intellectuelles: cependant cela n'arrive pas; les hommes different autant par les qualités de l'esprit que par les traits du visage. Il est, dans l'espèce humaine, des êtres aussi différents les uns des autres, que l'homme l'est ou d'un cheval ou d'un chien» (p. 133).

13. Réflexions sur l'existence de l'âme et sur l'existence de Dieu, dans G. Mori et A. Mothu (sous la dir. de), Philosophes sans Dieu. Textes athées clandestins $d u$ XVIII siècle, ouvr. cité, p. 271 : «Il est un axiome convenu, c'est qu'il ne faut pas multiplier les êtres sans nécessité. Si l'on conçoit que les opérations attribuées à l'esprit peuvent être l'ouvrage de la matière agissant par des ressorts inconnus, pourquoi imaginer un être inutile, et qui dès lors ne résout aucune difficulté?»

14. Voir les «Sentiments des philosophes sur la nature de l'âme», dans A. Mothu et A. Sandrier (sous la dir. de), Minora clandestina, I, Paris, Champion, 2003, p. 182: «Un philosophe du siècle dernier [Descartes] a expliqué plus particulièrement cette preuve par le raisonnement qui suit. Je pense et cette pensée n'est pas mon corps: cette pensée n'est ni longue, ni large, ni étendue, comme il est essentiel à la matière qui compose mon corps de l'être; elle n'est pas par conséquent sujette à la destruction comme lui; car la destruction ne peut se faire sans division de parties, et l'on ne peut concevoir de division de parties dans une substance qui n'a point d'étendue, telle que la pensée: il y a donc en moi, conclut ce philosophe, deux substances, l'une impérissable qui pense, et l'autre périssable qui ne pense point». 
consentement universel, ou sur l'existence d'autres êtres immatériels (génies, démons, substances aériennes), ou bien sur la noblesse spécifique de cette substance et de ses opérations témoignant ainsi de son caractère divin, ou bien encore sur la nécessaire rétribution ou punition post mortem des actions terrestres des êtres humains. Là encore, la solution est vite trouvée, qui consiste à dire que supposer l'âme matérielle permet de mieux expliquer les relations de réciprocité qu'elle entretient avec son corps, et que cette matérialité a pour conséquence nécessaire sa mortalité. En effet, comme l'indique les "Sentiments des philosophes sur la nature de l'âme», si le lien qui unit le corps à l'âme est extrêmement étroit, on doit en conclure que «ses fonctions se perfectionnent et s'affaiblissent selon l'accroissement ou la diminution de force dans les organes. Elles cessent totalement, et cet esprit de vie s'envole et se réunit au général, lorsque les dispositions qui le maintenaient dans le particulier viennent à cesser ${ }^{15}$ ».

Reste un point à éclaircir, celui qui concerne la thèse cartésienne de l'animal-machine évoquée dans de très nombreux manuscrits à titre de doctrine scandaleuse et absurde. À cet égard, les manuscrits se contentent souvent de piller Montaigne, Charron ou Bayle et d'alléguer: 1) qu'il existe une raison ou une intelligence animale certes différente de l'humaine mais néanmoins incontestable ${ }^{16}$, qui explique les vertus intellectuelles attribuables aux bêtes (fidélité, prudence, sociabilité, etc.) ; 2) que la différence entre homme et animal est de degré et non de nature et qu'elle consiste spécifiquement dans une organisation différente des organes, et en particulier du cerveau ${ }^{17}$; 3) que les animaux agissent en fonction

15. «Sentiments des philosophes sur la nature de l'âme», ouvr. cité, p. 192.

16. «Combien d'animaux font voir plus de douceur, de réflexion et de raison que l'animal qui se dit raisonnable par excellence! Est-il, parmi les hommes si souvent esclaves ou opprimés, des sociétés aussi bien constituées que celles des fourmis, des abeilles, des castors? » (Le bon sens du curé Meslier, ouvr. cité, p. 135).

17. «[...] toute la différence de la raison humaine à celle des animaux ne consiste que dans celle de l'organisation de leur cerveau, qui se trouve dans les hommes d'une disposition plus propre au raisonnement qu'il ne l'est dans les autres animaux» ( "Sentiments des philosophes sur la nature de l'âme», ouvr. cité, p. 187). «L'homme ne diffère des autres animaux que par la différence de son organisation, qui le met à portée de produire des effets dont ils ne sont point capables. La variété que l'on remarque entre les organes des individus de l'espèce humaine suffit pour nous expliquer les différences qui se trouvent entre eux pour les facultés que l'on nomme intellectuelles [...]. C'est par l'exercice, l'habitude, l'éducation que l'esprit humain se développe et parvient 
d'une fin et non uniquement par instinct. À l'égard de la doctrine spécifique de l'animal-machine, on retrouve souvent les deux mêmes arguments, l'un théorique, l'autre pratique. L'argument théorique se joue au niveau d'une extension de l'hypothèse du strict mécanisme animal appliqué à l'homme: il se pourrait bien que l'animal soit une machine admirable, mais, dans ce cas, l'homme pourrait bien être le chef-d'œuvre mécanique de Dieu, son automate le plus admirable dont la complexité expliquerait la présence en lui d'une pensée tout aussi déterminée que le sont les mouvements de son corps. On sait que Descartes s'était proposé cette objection, mais il l'avait rejetée comme moralement impossible. Une machine extrêmement complexe est bien envisageable, mais sa construction apparaît hautement problématique, car il faudrait la doter d'organes quasiment infinis pour lui permettre de faire face à toutes les situations dans lesquelles elle aura à se trouver. Et, parmi ces situations diverses, la machine aura à s'adapter aux conversations humaines, à leur extrême variabilité, ce qui paraît difficilement concevable. Pour l'auteur de L'âme matérielle, cette objection ne porte pas puisque la toute-puissance divine en fait une possibilité physique tout à fait acceptable ${ }^{18}$. La conséquence logique de la thèse de la réduction de l'animal à la machine est donc d'en arriver à la même conclusion pour l'homme. De son côté, l'argument pratique repose sur l'expérience et sert à montrer que le mécanisme à l'œuvre dans le corps animal n'explique pas toutes ses actions, sinon il serait impossible de rendre compte de comportements tels que celui d'un cheval refusant de se jeter dans un précipice alors même que son cavalier l'éperonne vivement pour qu'il aille de l'avant. Autre formulation qui permet de com-

à s'élever au-dessus des êtres qui l'environnent; l'homme sans culture et sans expérience est un être aussi dépourvu de raison et d'industrie que la brute» (Le bon sens du curé Meslier, ouvr. cité, p. 133-134). "Si toutes leurs actions [celles des hommes] ne procèdent que de la matière, pourquoi ne peut-on pas dire que l'âme de l'homme n'est qu'une matière modifiée de telle et telle façon, et que tout le bruit qu'il fait dans le monde, n'est que l'effet d'un corps mieux composé, et d'une machine plus parfaite: si les bêtes avaient l'usage de la langue et qu'elles pussent par cette faculté être instruites comme les hommes, elles feraient paraître à peu près les mêmes opérations " (L'âme matérielle, ouvr. cité, p. 118).

18. Voir la longue argumentation développée à ce propos dans L'âme matérielle (p. 100) qui s'inspire d'une recension de la Bibliothèque universelle et historique de 1693 (p. 132-134). Jacques Rohault avait déjà évoqué l'hypothèse de l'homme-machine dans ses Entretiens sur la philosophie, Paris, 1675, p. 149150. 
prendre que la différence entre l'espèce humaine et les autres espèces animales est de degré et non de nature.

Tous les arguments mentionnés précédemment pour réfuter le dualisme ontologique cartésien et le remplacer par un monisme matérialiste ne sont guère originaux et participent de ce combat responsable de la chute du cartésianisme à la fin du XVII siècle, déjà évoqué par Richard Watson ${ }^{19}$. Cela étant, la matérialisation de l'âme que proposent les manuscrits clandestins s'effectue selon deux modalités distinctes et normalement incompatibles, quoique parfois présentées de pair, à savoir un vitalisme et un animisme universels d'une part, et un mécanisme fonctionnel et organisationnel d'autre part, qui semble l'emporter au final en ce qui a trait à la description anatomique et physiologique du corps humain parce qu'il correspond mieux à la science de son époque. Ce réductionnisme mécaniste témoigne bien de l'influence du cartésianisme sur les manuscrits clandestins, même s'il s'agit de le travestir et de le retourner contre lui-même, et l'on comprend comment ce dernier, à travers la médiation clandestine, a pavé la voie menant à L'homme-machine de La Mettrie.

\section{Le cas spécifique des Réflexions morales et métaphysiques}

Au premier abord, le texte des Réflexions morales et métaphysiques sur les connaissances de l'homme ${ }^{20}$, dont nous connaissons l'auteur ${ }^{21}$, un certain Delaube, et la date approximative de rédaction (les premières années du XVIII ${ }^{e}$ siècle), s'inscrit dans la continuité de l'héritage du libertinage érudit. On y retrouve à longueur de pages les topoi de la littérature clandestine: la religion, bien que nécessaire pour maintenir l'ordre public, n'est qu'un instrument

19. Richard Watson, The Downfall of Cartesianism 1673-1712. A Study of Epistemological Issues in Late 17th Century Cartesianism, La Haye, Martinus Nijhoff, 1966, et The Breakdown of Cartesian Metaphysics, $2^{\mathrm{e}}$ édition, Indianapolis (Indiana) et Cambridge (Massachusetts), Hackett, 1998.

20. Il en existe deux manuscrits (Grenoble 329, Rouen 1569). Nous citerons toujours la copie de Grenoble en modernisant orthographe et ponctuation; le chiffre romain renvoie aux parties du traité, l'arabe au chapitre, et ces deux mentions seront suivies du numéro du feuillet auquel nous faisons référence. Nous remercions Antony McKenna de nous avoir fourni une copie de ce manuscrit.

21. Voir Miguel Benítez, «Liber ebendus de religione abolenda: réflexions morales et métaphysiques sur les religions et sur les connaissances de l'homme», Lias, Amsterdam, vol. 17, nº 2, 1991, p. 163-184. 
aux mains des politiques pour abuser le peuple; le vulgaire est toujours crédule et prompt à se laisser berner par les prêtres; la véritable libération n'est possible que pour des esprits déniaisés, qui sont parvenus à une compréhension exacte de la nature humaine; les hommes ne sont guidés que par leur amour-propre et le goût de la luxure (ce qui explique les nombreuses condamnations du libertinage de mœurs); le péché originel est un dogme sans consistance qui détourne de la vraie connaissance du cour humain; les miracles ne tiennent pas face à une critique sérieuse de la valeur des témoignages; les êtres naturels sont tous égaux et l'être humain ne peut se prévaloir d'aucune supériorité, etc. Le plan de l'ouvrage ne laisse d'ailleurs aucun doute sur la nature de cet héritage: il faut en premier lieu se libérer des préjugés de l'enfance, ceux qui concernent Dieu ou la religion dont on nous a fourni des images quand notre raison ne pouvait encore les passer au crible, puis comprendre la nature en général telle qu'elle se présente à nous, et plus spécifiquement la nature humaine, et ce afin de dégager un certain nombre de principes permettant de jouir au mieux de l'existence terrestre.

S'il s'inscrit bien dans une perspective assez commune à l'âge classique, ce manuscrit n'en est pas moins original à un autre niveau. Son véritable intérêt réside dans la métaphysique qu'il met en ouvre pour asseoir cette éthique censée procurer à ses lecteurs la paix de l'esprit et du corps, et un bonheur terrestre libéré de toute crainte ou espérance quant à la vie future. Adoptant une démarche cartésienne qui se fonde sur le recours à la raison, faculté naturelle égale en tout homme, et qui vise à se départir des préjugés de l'enfance, Delaube s'engage à son tour dans des méditations métaphysiques qui le conduisent d'entrée de jeu à un refus du scepticisme, ce "pyrrhonisme qui fait profession de douter de tout ${ }^{22} »$ (préface, 3 ), et à une double conviction, celle de l'existence de Dieu et celle de l'existence du moi. Les arguments apportés pour parvenir à une telle conclusion sont repris de Descartes, parfois quasiment au mot près, même si des différences essentielles persistent, notamment à l'égard de l'essence du moi :

22. Voir aussi le passage suivant où le refus du scepticisme est tout aussi clair: «j'ai bien quelque connaissance de ce que je dis car je n'aime à suivre ni la superstition qui croit tout, ni le pyrrhonisme qui ne croit rien; croire tout est au-dessus de la raison et ne croire rien est au-dessous; la superstition rend un homme fou, et le pyrrhonisme en fait un furieux: je cherche un juste milieu » $(\mathrm{I}, 8,121)$. 
Si j'étais l'auteur de moi-même, je verrais clairement ma nature. La conviction où je suis de ma faiblesse et de ma profonde ignorance me persuadent clairement que je ne suis pas l'auteur de mon être. Que dis-je? Il faut être insensé pour être entré dans ce doute, car, pour me produire, il faudrait exister auparavant. Et si j'étais alors, je ne m'étais donc pas fait, il n'était pas même nécessaire de me produire - cette demande est ridicule et chimérique. Cependant je me sens, je raisonne, je ne saurais sentir ni penser sans être, je pense donc je suis, et sans me mettre plus en peine de savoir ce que je suis, ce qui est impossible, il suffit que je sois convaincu de mon existence pour être obligé d'en rechercher l'auteur. Je vois bien qu'il faut sortir de chez moi pour être en état de faire cette découverte (II, 1, 7-8).

La suite du texte roule sur la possibilité que le moi soit le fruit du hasard et conclut, après une réfutation en règle de cette hypothèse, à la seule solution qui paraisse vraisemblable, celle d'un Dieu unique conçu comme auteur de l'existence de ce moi. Seulement, cette double conviction de l'existence de Dieu et du moi, que l'on verra réapparaître de manière constante tout au long du manuscrit, n'épuise pas pour autant ses objets, la nature de Dieu ou du moi restant toujours inconnue. Un premier écart avec la démarche cartésienne se produit à ce niveau, puisque l'essence du moi ne peut être aperçue de manière intuitive comme équivalente à la pensée, seule son existence étant appréhendée avec évidence. Malgré tout, le chemin emprunté par Delaube paraît encore proche de celui déjà parcouru par Descartes. La suite le confirme, puisque le manuscrit en vient également à montrer toute la difficulté de prouver l'existence des corps extérieurs ${ }^{23}$. Seulement, là où Descartes postulait un Dieu vérace pour résoudre la question de cette existence problématique, Delaube préfère négliger une telle réponse qui ne lui paraît pas cohérente et quitte alors la voie pavée par Descartes pour suivre des sentiers déjà balisés par Malebranche et l'abbé de Lanion, et qui seront bientôt empruntés par Collier ou Berkeley ${ }^{24}$.

23. «Niez l'existence des corps en présence d'un physicien, ou d'un médecin, vous serez regardé comme un homme qui n'a pas le sens commun, ce n'est pas qu'ils soient parfaitement convaincus de l'existence de la matière, car il faut de puissantes preuves pour former cette conviction entière, je puis même avancer qu'il n'en est point» (préface, 6-7).

24. De nombreux passages du manuscrit sont spécifiquement anti-cartésiens, comme nous l'avons montré dans "L'immatérialisme dans la littérature clandestine du siècle des Lumières ", Dialogue, Waterloo (Ontario), vol. 39, $\mathrm{n}^{\circ} 1,2000$, p. 491-511. 
Ainsi, au lieu de se diriger, avec la grande majorité des autres manuscrits clandestins, vers une matérialisation de l'âme, Delaube prend la direction inverse et argumente en faveur d'une spiritualisation de la matière qui rappelle certaines intuitions "égoïstes" selon lesquelles l'âme représente le seul être avéré ${ }^{25}$. Outre le fait que Delaube prétende qu'il ne dispose d'aucune preuve solide en faveur de l'existence de la matière et que l'esprit seul soit actif, le manuscrit adopte une position idéaliste forte qui récuse l'existence de la matière et qui réduit les qualités primaires aux secondes. La conséquence d'une telle réduction conduit Delaube à prêcher une forme de subjectivisme qui s'apparente à un idéalisme proche du solipsisme:

C'est l'esprit qui sent, voit et entend, on n'en peut pas douter. Cela étant, tous les objets visibles et sensibles sont dans l'esprit; il n'est dans l'âme que deux opérations qui sont la pensée et le sentiment, tout se réduit là quoi qu'elle fasse. Il est visible que toutes les facultés des sens sont comprises dans la seconde opération, voir, goûter, entendre, toucher, etc. Il s'ensuit de là que quand elle voit, ou entend, elle le sent; elle ne saurait sentir que dans elle-même, donc elle voit et entend dans elle-même, puisque voir et entendre n'est que sentir; donc tous les objets sensibles et visibles sont dans l'âme, attendu qu'ils en sont des sensations (I, 2, 32).

Les objets ne sont rien d'autre que l'âme modifiée et n'existent en elle qu'en tant qu'ils sont perçus. S'ils en étaient distincts réellement, elle ne pourrait pas les sentir. Il s'agirait ici d'un véritable solipsisme si l'esprit était le seul être percevant, ce qui n'est pas le cas puisque Dieu est également présent, sous la forme d'une divinité omnipercevante que Berkeley n'aurait pas récusée: «La divinité ne voit pas les êtres ni leurs manifestations parce qu'ils subsistent, si cela était ils devraient précéder sa vue, mais ils ne subsistent que parce qu'elle les voit, il y a un rapport essentiel entre la vision d'un Dieu et l'existence d'un objet» (II, 3, 31).

Mais les rapprochements s'arrêtent là. Au niveau théologique, ses critiques du christianisme, sa remise en question de la liberté humaine, sa volonté de parvenir à l'être suprême sans médiation religieuse, sont des éléments qui l'éloignent de la position plus conservatrice de Berkeley. Au niveau philosophique, Delaube est plus un héritier des débats cartésiens que celui qui ouvre la voie à

25. Sur tout ceci, voir toute la première partie de notre Berkeley au siècle des Lumières. Immatérialisme et scepticisme au XVIII' siècle, Paris, Vrin, 2003. 
un immatérialisme de type berkeleyen. C'est d'ailleurs sans doute au sein de ces débats qu'il a puisé sa métaphysique qui l'éloigne définitivement d'un immatérialisme tel que celui qui sera développé par Berkeley quelques années plus tard et le conduit à une forme de scepticisme épistémologique qui le fait hésiter entre un dualisme strict et un monisme spiritualiste conséquent:

La vue de tous ces objets visibles que j'appelle soleils, terre, planètes, hommes, animaux, fait-elle partie de ma substance, ou est-elle distincte de ma personne? Si j'interroge mon esprit, il m'apprend qu'ils ne sont que des sensations de lui-même, les sens me disent le contraire. Comment comprendre que tout cet univers sensible ne soit que mon esprit modifié, ou qu'il en soit distinct? L'un et l'autre sentiment sont également incompréhensibles, et font voir que nous portons un fonds d'ignorance incapable de rien nous apprendre de clair sur la nature de ce monde qui sera toujours pour nous une énigme impénétrable (I, 3, 43-44).

Le leitmotiv du manuscrit est bien la reconnaissance de cette ignorance totale de la nature de l'esprit humain et du monde qui l'entoure. Au niveau de la perception seule du rapport entre l'esprit et le corps, Delaube paraît se rapprocher de Descartes et admettre que le moi qui pense et qui sent, dont la spiritualité implique l'unicité, est différent du moi corporel qui, lui, est composé de parties, parties qui peuvent être retranchées du corps sans que pour autant la force du principe spirituel en soit diminuée. L'esprit pourrait bien être réductible au cerveau malgré tout, et la mort cérébrale correspondre à la fin de l'existence, mais cette proposition, sans être réfutée, paraît aller contre le bon sens. Les dernières lignes du manuscrit reviendront sur cette question, là encore sans trancher, mais en laissant entendre que la réincarnation ou le retour en Dieu l'emportent en termes de vraisemblance sur la destruction totale de l'individu.

La question du rapport entre âme et corps est paradigmatique de la méthode de Delaube qui, sur chaque thèse métaphysique centrale, oppose idéalisme et matérialisme, à l'avantage de l'idéalisme qui paraît plus vraisemblable, sans pour autant trancher sur le fond puisque, selon ses principes mêmes, une conclusion définitive sur ces questions est de l'ordre de l'impossible. Ainsi, dans le cas de l'existence réelle de la matière, c'est encore une fois l'hypothèse idéaliste qui paraît la plus vraisemblable. Cela se comprend simplement par le fait que rendre la matière indépendante de Dieu et donc réellement agissante reviendrait à en faire un être 
indépendant et égal à Dieu, ce qui serait absurde. Mais la rendre dépendante de Dieu serait tout aussi absurde, puisque cela compliquerait à l'envi le modèle et ferait de Dieu un architecte brouillon à la toute-puissance bien peu efficace. Nous ne dépendons que de Dieu, et c'est pourquoi la matière n'existe pas, car si elle existait, nous en serions dépendants. Nous ne pouvons donc habiter un corps matériel qui aurait été créé pour recevoir notre esprit et qui dépendrait en quelque façon de lui. Autre argument: si le contenant est ce qui enveloppe le contenu et lui est supérieur, alors la matière, qui enveloppe et affecte l'esprit, serait supérieure à ce dernier, ce qui est absurde. Le seul contenant réel est Dieu et c'est de lui seul que nous dépendons. Le corps visible est une portion de la divinité qui n'appartient pas à l'esprit auquel il est lié, il s'agit d'une machine qui fonctionne selon des lois fixées par Dieu.

Un argument emprunté à l'abbé de Lanion pousse Delaube du côté de l'idéalisme, celui de la simplicité des voies ${ }^{26}$. Si Dieu peut produire dans l'esprit humain les mêmes affections avec ou sans la matière, pourquoi donc aurait-il besoin de cette dernière? Ce serait emprunter un détour inutile, que les philosophes ne conservent que pour des raisons de cohérence, en croyant justifier l'action divine par l'existence des corps. Mieux vaut penser que Dieu a agi de la manière la plus économe possible: "On ne doit pas multiplier les êtres sans nécessité. Il n'est point de milieu: tout est Dieu et esprit, les esprits sont en Dieu, et ce monde visible n'est qu'un faible rayon de la divinité» (II, 9, 164-165).

L'hypothèse idéaliste apparaît donc comme la plus probable, «car il y a bien plutôt apparence que ce monde visible n'est que cet esprit universel qui nous affecte tous, et qui se manifeste diversement à nous du côté seulement de son immensité» $(\mathrm{I}, 8,111)$. On semble ici bien proche de Malebranche, si ce n'est que chez ce dernier l'existence des corps est rendue compréhensible par la médiation du texte révélé. Pour Delaube, qui ne croit pas à la révélation, elle reste toujours problématique. Mais cette ignorance de l'intérieur absolu de ce qui constitue le monde n'est que de peu d'importance. Bien sûr, les questionnements sur l'essence des

26. Lanion, Méditations sur la métaphysique, $\mathrm{VI}^{\mathrm{e}}$ Méditation: «Dieu qui agit toujours par les voies les plus simples, étant la cause immédiate de mes modifications, j'ai sujet de penser que ce serait un trop long détour à cet être infiniment sage, que de créer de l'étendue pour me donner des sensations qu'elle ne peut pas produire» (cité par François Pillon, "L'idéalisme de Lanion", L'Année philosophique, Paris, 6e année, 1895, p. 126). 
choses sont essentiels à la démarche métaphysique et conduisent à deux ordres d'explication généraux, matérialiste ou idéaliste, à propos desquels on ne peut trancher. En outre, ces questionnements paraissent sans fin et touchent tous les niveaux du vivant comme le montre le débat sur la nature de l'animal, qui pourrait bien être une machine ${ }^{27}$, ou celle des plantes, qui pourraient bien être dépourvues de toute sensation. Toute réponse à cet égard est de l'ordre du plausible, Delaube choisissant pour sa part une interprétation combinant idéalisme et panthéisme qui fait de l'univers un immense vivant rempli de mondes habités, dont la cohérence et la survie s'expliquent par la Providence divine qui se manifeste selon des lois universelles et nécessaires. Reprenant l'exemple de Malebranche, il montre que la pluie est utile même dans les déserts les plus secs et que l'action divine se déploie sous la forme de lois générales, uniformes et variées à la fois, qui favorisent tous les êtres vivants sans distinction aucune. Ainsi la pluie tombe-t-elle partout, mais en quantité variable et à des périodes différentes. On le voit bien, qu'il s'agisse de la nature du monde, de l'essence de l'esprit, de la raison animale ou de la sensation végétale, toutes ces questions ne peuvent être résolues une fois pour toutes.

Les seules certitudes que la raison procure sont donc celles de l'existence de Dieu et du moi, et de la relation de dépendance existant entre elles. Si l'on fait un retour sur soi et que l'on cherche à comprendre quelle est la nature de la divinité, un certain nombre d'attributs semblent lui appartenir de plein droit: l'éternité, la nécessité, l'immensité et l'immuabilité. Reste à comprendre quelle est la nature de ces différents attributs et ce qu'elle implique. C'est peut-être là que le panthéisme de Delaube se comprend le mieux.

27. Mais ce choix de réduire l'animal à la machine est dangereux et peut conduire à une réduction identique pour l'homme: «Si les animaux dans leur conduite admirable n'agissent que machinalement, pourquoi les hommes n'agiront-ils pas de même? Les pensées et les sentiments qu'ils croient avoir sont-ils différents du corps? Ils assurent l'affirmative, mais ils disent cela gratis, qu'en savent-ils dans le fond? Ne sait-on pas que tous les efforts qu'ils produisent pour le prouver ne font que blanchir là-dessus? Peut-être que leurs opérations prétendues spirituelles ne sont autre chose que des parties subtiles du cerveau, ainsi qu'on le veut dans les animaux. Car puisque ce principe prétendu spirituel est dans le fond inconnu et qu'on peut d'ailleurs concevoir encore plus aisément la machine bipédique capable des mêmes opérations indépendamment de ce principe, pourquoi ne dirons-nous pas aussi qu'ils sont avec le reste des animaux de pures machines? Voilà où conduit le raisonnement des hommes qui veulent ôter aux animaux tout principe distinct et doué de connaissances» (I, 7, 94-95). 
Dieu est éternel en ce sens qu'il est toujours présent et que le monde entier existe dans cette présence, ce qui rend l'univers éternel. Bien plus, tous les êtres sont co-éternels à l'essence divine, même s'ils n'en ont pas nécessairement conscience.

Ce n'est pas par mes pensées et mes sentiments successifs que je juge de mon éternité, c'est par l'immuabilité de la vision divine, en présence de laquelle toute la propriété des objets demeure dévoilée et immuable, et dont les créatures ne s'aperçoivent que successivement à cause des bornes étroites de leur capacité, de sorte que notre éternité ne consiste que dans une révolution continuelle de vies différentes dont nous perdons le souvenir par la perte de notre machine, et par la succession d'une nouvelle où nous commençons une autre vie. Ainsi nous volons pour ainsi dire de monde en monde et nous nageons éternellement dans l'immensité divine (II, 2, 23).

Le rapport entre Dieu et le monde doit se comprendre selon le modèle spinoziste d'une distinction entre nature naturante et nature naturée. Dieu est l'être réellement agissant, puissance pure, qui voit de toute éternité les choses en elles-mêmes dans l'étendue de leur perfection et non à titre d'archétypes ou de modèles, choses présentes dans son immensité et pourtant distinctes, que ce soit au niveau des actions ou des pensées humaines. Si Dieu est l'être pleinement actif, si tout est sous la dépendance de son action nécessaire, alors le libre arbitre, comme l'avait vu également Spinoza, n'est rien d'autre qu'une illusion ${ }^{28}$. Si nous avions disposé d'une telle liberté, Dieu nous aurait permis d'aller contre sa volonté, ce qui est absurde. Dieu seul est libre, mais d'une liberté à entendre en termes spinozistes, tous les autres esprits étant dépendants des corps qu'ils occupent, des passions qui les meuvent et des perceptions qu'ils reçoivent du monde extérieur. Cette conception d'un Dieu nécessaire aux décrets immuables rend superflues toutes les religions parce qu'elles reposent sur l'hypothèse d'une liberté humaine effective.

Ce déterminisme absolu pose évidemment problème. Si tous les êtres naturels sont déterminés par une loi unique qui est celle

28. «Il est constant que je suis votre ouvrage, mon Dieu, que vous avez formé en moi une infinité de passions qui m'agitent, et dont le mouvement fait toute ma vie. Le reste de ce mouvement m'est inconnu, et il est devant vous, toutes mes inclinations vous sont réellement présentes, elles ne se développent en moi que les unes après les autres; à chaque instant je ne fais que remplir ma destinée, c'est pour dire un développement de mon sort» (II, 3, 32). 
de l'amour-propre, qui correspond en gros au conatus spinoziste, c'est-à-dire à la conservation de soi, comment peut-on parvenir à une forme réelle de libération et à un dépassement des préjugés de l'enfance? À force de trop vouloir dénier toute part de liberté aux hommes pour critiquer l'anthropomorphisme des religions, Delaube est quasiment conduit à nier toute activité humaine réelle:

Dieu a bien donné aux bipèdes un pouvoir d'agir qui n'est rien dans le fond de réel et de distinct de Dieu même, ce n'est jamais que la volonté divine qui pousse et anime notre esprit et qui nous fait apercevoir successivement de nos différentes sensations qui paraissent éternellement en présence de son immensité. Notre volonté ou notre esprit abandonné de la volonté divine se trouve sans force et sans mouvement; il est semblable à l'instrument d'un ouvrier dont la main lui donne toute la force (II, 5, 84).

Or, comment promouvoir une éthique, objectif principal des Réflexions morales et métaphysiques sur les connaissances de l'homme, sans reconnaître aux hommes une part minimale de liberté? Comment promettre le bonheur si ceux-ci ne peuvent choisir la voie de leur salut terrestre? Pourquoi dresser un tableau de l'honnête homme si cette honnêteté n'est pas le fruit d'un travail sur soi, mais le simple résultat de l'ordonnancement divin? Sur cette question, Delaube reste vague. La libération lui apparaît comme une chose exceptionnelle, car l'ordre général du monde implique que les religions ne disparaissent pas — en apportant une consolation aux pauvres et aux démunis et en réprimant les crimes par la crainte de châtiments éternels, elles sont le garant de l'ordre civil - et donc qu'il n'y ait qu'un petit nombre de déniaisés ${ }^{29}$. Mais cette libération devrait être d'autant plus exceptionnelle que Delaube la décrit sous la forme d'une maîtrise des passions, ce qui semble quelque peu contradictoire avec sa conception du déterminisme intégral où libre arbitre mais aussi liberté paraissent illusoires et où une telle libération semble bien n'être le fait que de la volonté de Dieu seul qui choisit ou non d'éclairer certains individus au détriment d'autres qu'il laisse dans l'ignorance la plus crasse.

29. «Le commun des bipèdes n'est pas capable de prendre un essor si élevé, ils sont destinés à ramper et à subir le joug éternellement, cela est nécessaire pour l'usage des religions, lequel est essentiel à la conservation des sociétés » (II, 6, 115-116). 
Sur cette question de la liberté et de la libération possible, Delaube n'est pas toujours cohérent dans la concordance entre sa métaphysique et son éthique. Mais c'est que, pour lui, l'essentiel se joue ailleurs, au niveau des actions que l'on doit entreprendre au quotidien et des motivations qui les guident. Certes, en dernier recours, son éthique est indissociable d'une conception métaphysique du monde, mais elle ne doit pas être totalement subordonnée aux résultats de cette dernière. Sinon, ce serait donner toute la place aux spéculations métaphysiques, qui ne déboucheront d'ailleurs jamais sur une connaissance absolue et véridique des choses, et en oublier l'important, qui est de vivre, et du mieux possible. Cela implique de rompre avec les préjugés en usant d'une raison certes faible mais non inefficace et de vivre dans une tranquillité d'esprit propre au bonheur, sans craindre la mort à venir. La voie de la sagesse est celle de la maîtrise des passions qui vise la santé de l'âme et du corps, et qui seule procure la joie parfaite et fait de ce monde un paradis, le seul possible ${ }^{30}$. Pour ceux qui ne parviendront pas à cette maîtrise de soi et vivront dans l'excès (les libertins débauchés, les fanatiques, les ambitieux), ce monde prendra le visage d'un enfer.

L'intérêt de ce manuscrit très particulier que sont les Réflexions morales et métaphysiques sur les connaissances de l'homme ne concerne pas l'éthique proposée, mais la métaphysique retenue. Cette métaphysique qui mêle adroitement idéalisme et panthéisme montre que la matérialisation de l'âme n'était pas la seule avenue possible dans un contexte fortement marqué par le cartésianisme. Mais s'il s'inspire de la conception dualiste de Descartes, Delaube refuse lui aussi de destituer les bêtes de sentiment et de raison et penche en faveur d'un vitalisme universel au nom de la toutepuissance divine, là où les matérialistes se divisent en partisans d'un animisme universel hérité de la Renaissance ou d'un matérialisme nécessitariste inspiré du mécanisme cartésien ${ }^{31}$. Cet anticartésianisme vu à travers le prisme de la philosophie clandestine permet d'interpréter différemment l'inflexion subie par la pensée de Descartes à la fin du XVII ${ }^{e}$ siècle et au début du XVIII ${ }^{e}$ siècle sous la double forme d'une continuation et d'une rupture. Ce double

30. «Nous sommes en Dieu et par conséquent dans un paradis éternel; il n’en est point d'autre, celui qu'on imagine est un fantôme» (II, 10, 186-187).

31. Voir Aram Vartanian, "Quelques réflexions sur le concept d'âme dans la littérature clandestine», dans Olivier Bloch (sous la dir. de), Le matérialisme du XVIII siècle et la littérature clandestine, Paris, Vrin, 1982, p. 149-163. 
mouvement s'explique par l'impossibilité de résoudre dans les termes mêmes de Descartes la question du rapport entre l'âme et le corps. Pour certains partisans du dualisme cartésien, il faut dépasser le maître en poussant l'idéalisme à son terme et se résoudre à une forme d'immatérialisme s'il le faut. Pour leurs adversaires, il s'agit de verser à l'opposé dans un monisme matérialiste qui permet seul d'expliquer l'interaction de la pensée et de l'étendue. Cela étant, matérialistes et idéalistes ont au moins une chose en commun, l'impression de n'avoir fait que tirer les conséquences logiques de la métaphysique de Descartes, quitte à en déformer le message et à en manquer parfois la subtilité. 